\title{
Non-parametric linear time-invariant system identification by discrete wavelet transforms
}

\author{
R.W.-P. Luk ${ }^{\mathrm{a}}$, R.I. Damper ${ }^{\mathrm{b}, *}$ \\ a Department of Computing, Hong Kong Polytechnic University, Hung Hom, Kowloon, Hong Kong \\ ${ }^{\mathrm{b}}$ Image, Speech and Intelligent Systems (ISIS) Research Group, School of Electronics and Computer Science, University of Southampton, \\ Southampton, SO17 1BJ, UK
}

Available online 9 December 2005

\begin{abstract}
We describe the use of the discrete wavelet transform (DWT) for non-parametric linear time-invariant system identification. Identification is achieved by using a test excitation to the system under test (SUT) that also acts as the analyzing function for the DWT of the SUT's output, so as to recover the impulse response. The method uses as excitation any signal that gives an orthogonal inner product in the DWT at some step size (that cannot be 1). We favor wavelet scaling coefficients as excitations, with a step size of 2. However, the system impulse or frequency response can then only be estimated at half the available number of points of the sampled output sequence, introducing a multirate problem that means we have to 'oversample' the SUT output. The method has several advantages over existing techniques, e.g., it uses a simple, easy to generate excitation, and avoids the singularity problems and the (unbounded) accumulation of round-off errors that can occur with standard techniques. In extensive simulations, identification of a variety of finite and infinite impulse response systems is shown to be considerably better than with conventional system identification methods.
\end{abstract}

(c) 2005 Elsevier Inc. All rights reserved.

Keywords: System identification; Linear-time invariant systems; Discrete wavelet transform

\section{Introduction}

Wavelet analysis [1-4] provides a unifying framework for time-frequency decomposition of signals. It has found important applications in compression [5], denoising [6], transient signal detection [7], adaptive filtering [8,9], channel equalization [10], identification of echo path impulse responses [11], and modeling mammalian auditory system function [12,13]. It has a direct correspondence to filterbank analysis [14]. Here, we consider wavelet approaches to analyze signals that are a (linearly) filtered version of some source signal with the purpose of identifying the characteristics of the filtering system. Such source-filter signals occur in many physical situations. A well-known example is the human speech production mechanism where air waves modulated as a sequence of (quasi-)periodic pulses at the larynx are filtered by the vocal tract. In this particular case of a biological system, the input to the system is not accessible to the investigator. For many engineering systems, however, we do have reasonable accessibility. In these

\footnotetext{
* Corresponding author.

E-mail addresses: csrluk@comp.polyu.edu.hk (R.W.-P. Luk), rid@ecs.soton.ac.uk (R.I. Damper).
} 
circumstances, the source-filter model lends itself directly to the extremely important practical problem of finding the characteristics of a system under test (SUT).

System identification methods can be classified as parametric and non-parametric approaches. By 'parametric,' we mean that the functional form of the system model is known but its parameters (e.g., specifying the location of poles and zeros in the complex plane) are not. By 'non-parametric,' we mean that this functional form is unknown so that the SUT is alternatively described explicitly by its output for some given wideband input. In principle, we could use any wideband input for this purpose but, in practice, the approach is only useful if we standardize on one of a very few functions, such as unit step or impulse, to avoid a plethora of incommensurate measures and descriptions. In the discrete-time case, this description will be a set of sample values. Identification is further divided into time-domain and transformed-domain techniques.

In the (continuous) time domain, the most straightforward technique from a theoretical perspective is to excite the SUT with a Dirac impulse, whereupon the output is the impulse response function $h()$-hence its name. Although theoretically attractive, this is an impractical idealization for a real, physical system. The Dirac impulse ('delta function') is not truly a function at all, but a 'unit mass' abstraction [15, p. 5]: it has infinite amplitude at the point at which its argument is zero, is infinitely narrow and has unity integral over time. In the discrete-time case, we can attempt to approximate this abstraction by an input that changes amplitude entirely within one sampling period, i.e., by a Kronecker delta appropriately scaled in amplitude. In practice, however, this approximation is unlikely ever to be entirely satisfactory. Hence, other wideband input excitations (e.g., bandlimited white noise, frequency chirp) are sometimes used.

To avoid such difficulties, assuming a causal system, the impulse response function of the SUT can be recovered from the (sampled) output signal $\{y(n)\}$ for a (sampled) input signal $\{x(n)\}$ of any general form by the following recursive equation [16], obtained directly from the convolution-sum:

$$
h(n)=\frac{y(n)-\sum_{k=1}^{n-1} h(k) x(n-k)}{x(0)} \quad \text { for } n \geqslant 0, n \in \mathbb{N} .
$$

However, round-off errors accumulate with larger time indices, making this approach impractical for slowly decaying (i.e., infinite) impulse response functions.

The transformed-domain approach simply takes the $z$-transform of the output signal as $Y(z)=H(z) X(z)$ and determines the SUT impulse response function by inverse filtering the output signal by the input signal as $H(z)=$ $Y(z) X^{-1}(z)$. Unfortunately, the inverse of $X(z)$ does not always exist, so that it is necessary to use the pseudoinverse. Even then, the inversion operation may lead to an unstable inverse filter with no unique realization.

Two popular and inter-related frequency-domain methods for non-parametric system identification are based on coherence analysis. For a linear system, the coherence function [17] is given as

$$
C_{x y}\left(\omega_{k}\right)=\frac{S_{x y}\left(\omega_{k}\right)}{\sqrt{S_{x x}\left(\omega_{k}\right) S_{y y}\left(\omega_{k}\right)}},
$$

where $S_{x y}\left(\omega_{k}\right)$ is the input-output cross-spectrum (i.e., the power spectrum of the cross-correlation between the input and output functions), and $S_{x x}\left(\omega_{k}\right)$ and $S_{y y}\left(\omega_{k}\right)$ are the power spectra of the autocorrelations of the input and output, respectively. The function $C_{x y}^{2}\left(\omega_{k}\right)$ can be interpreted as the fraction of the mean square value of $y(n)$ that can be attributed to the component of the input $x(n)$ at frequency $\omega_{k}$. Usually, pseudorandom noise is used an input $x(n)$. The two identification methods, direct and inverse, then estimate the system response as

$$
\begin{aligned}
H_{1}\left(\omega_{k}\right) & \sim \frac{S_{x y}\left(\omega_{k}\right)}{S_{x x}\left(\omega_{k}\right)}, \\
H_{2}\left(\omega_{k}\right) & \sim \frac{S_{y y}\left(\omega_{k}\right)}{S_{x y}\left(\omega_{k}\right)},
\end{aligned}
$$

where $H_{1}\left(\omega_{k}\right)$ tends to underestimate the true $H\left(\omega_{k}\right)$ and $H_{2}\left(\omega_{k}\right)$ tends to overestimate it. Generally, $H_{1}\left(\omega_{k}\right)$ gives a good estimate of the system response near anti-resonances but $H_{2}\left(\omega_{k}\right)$ gives maximal error near anti-resonances. Conversely, $H_{2}\left(\omega_{k}\right)$ gives a good estimate of the system response near resonances whereas $H_{1}\left(\omega_{k}\right)$ gives maximal error near resonances.

By contrast, the parametric approach assumes the functional form of the system response is known and finds the parameters of this function, usually expressed as poles and zeros. Identification is achieved by iteratively minimizing 
the output error of the system according to the parametric model. Output error can be measured in the (stochastic) mean square sense, or maximum likelihood sense. Although parametric descriptions are more parsimonious than their non-parametric counterparts, the order of the model description has to be predefined but its proper choice is uncertain. If errors are required to be very small, large numbers of poles and zeros may be required.

Wavelet transforms have been extensively applied to non-linear system identification [18-23], as well as to parametric and/or time-varying system identification [19,24-27]. This is usually achieved by using a wavelet-based adaptive filter [28] and by non-linear regression [18] to perform a parametric identification, with a specified number of poles and zeros. For non-parametric identification, there has been little work, particularly for time-invariant signals, because discrete wavelet transforms (DWTs) are not time-invariant [4]. Since time-invariant systems and signals form a large well-known class, DWTs have been modified to be time-invariant $[29,30]$ but there is no clear relation between time-invariant signals and discrete wavelet transforms in general. Here, we examine the non-parametric identification of linear time-invariant (LTI) systems using the DWT. In particular, we show that the DWT of an output signal from an LTI system excited by the particular (mother) wavelet corresponding to the chosen transform is the impulse response of that system, and we use this result to develop a new method of system identification.

The rest of this paper is organized as follows. In Section 2, we review the discrete parameter wavelet transform for the continuous-time case and make an important observation about the orthogonality of the inner product between two identical wavelets. In Section 3, the discrete parameter wavelet transform is applied to a source-filter (i.e., output) signal of a linear time-invariant system, and the transform coefficients are found to relate directly to the impulse response of the system. For computer processing, the discrete-time wavelet transform (DWT) is also specified (Section 4). In Section 5, we develop a DWT-based technique for system identification that requires orthogonality of inner products computed by the DWT. In Section 6, various simulations are described to illustrate the use of the method for LTI system identification for different system types. In Section 7, we compare the identification performance of the method with conventional techniques. In Section 8, we summarize and conclude.

\section{Wavelet representation of signals}

A finite energy signal $f(t)$ in the square integral sense, i.e., $f(t) \in L^{2}$ (), can be described ('synthesized') by wavelets as

$$
f(t)=\sum_{k, m} a_{k, m} \psi_{k, m}(t), \quad k, m \in \mathbb{Z}
$$

where the set of two-dimensional coefficients $a_{k, m}$ is called the discrete parameter wavelet transform (DPWT) of $f(t)$ and the (continuous time) $\psi_{k, m}(t)$ 's are the analyzing functions, or wavelets, with scale index $k$ compressing/dilating the basic function, or mother wavelet $\psi_{0,0}(t)$, and translation index $m$ displacing it, to produce a family of wavelets. Although not strictly necessary from a theoretical perspective, in practical cases these wavelets are limited in time. They either have compact time support or rapidly decay to become close to zero, approximating compact time support.

In the DPWT, a wavelet is scaled and translated relative to the mother wavelet by discrete values $(k, m)$. This can be seen as a sampled counterpart to the continuous wavelet transform in which the scale and translation variables are continuous. Most often, compression/dilation in the DPWT is by a power of two-so-called dyadic sampling. That is, the wavelets are of the form $\psi\left(2^{k} t+m\right)$, with $\psi_{0,0}(t)=\psi(t)$.

Each DPWT coefficient, $a_{k, m}$ in Eq. (3), is simply computed as an inner product of the signal and the corresponding wavelet via the 'analysis' equation:

$$
a_{k, m}=\int f(t) \psi_{k, m}(t) d t=\left\langle f(t), \psi_{k, m}(t)\right\rangle
$$

If the wavelets are orthogonal, then

$$
\left\langle\psi_{k, m}(t), \psi_{r, s}(t)\right\rangle=C \delta(k-r) \delta(m-s), \quad k, m, r, s \in \mathbb{Z},
$$

where $C$ is a constant and $\delta()$ is the Kronecker delta, equal to 1 when its argument is zero and equal to 0 otherwise. Hence, we obtain only a single non-zero inner product (for the case $k=r$ and $m=s$ ) when the scale and translation indices range over all possible values. 
An interesting observation arises if the signal $f(t)$ has the same functional form as the analyzing (mother) wavelet, i.e., $f(t)=\psi_{\alpha, \beta}(t)$. Then, by Eq. (4), the DPWT of $f(t)$ has coefficients:

$$
a_{k, m}=\left\langle\psi_{\alpha, \beta}(t), \psi_{k, m}(t)\right\rangle \text {. }
$$

If the family of wavelets is orthogonal, then, by Eq. (5), $a_{k, m}$ will be non-zero only if $\alpha=k$ and $\beta=m$. The latter condition is easily achieved since both are indices relative to the same (sampled) underlying time scale (i.e., there exists some $m=\beta$ ). The former condition is slightly more problematic since there is no restriction on the scale of the signal $f(t)$. Therefore, for orthogonal wavelets, the scale index $\alpha$ should be well chosen to coincide with $k$ so that the coefficients do not vanish.

Analysis of the signal as just described corresponds to a multiresolution decomposition of a particular form that under certain conditions allows perfect reconstruction of the signal. Specifically, the decomposition involves taking an inner product of the signal $f(t)$ with a 'scaling' or 'dilation' function and sampling the result to produce a discretetime sequence $f(n)$, followed by successive splitting of the signal into subbands using non-overlapping high-pass and low-pass filters, $\bar{h}(n)$ and $\bar{g}(n)$ respectively, decimating each output sequence sample rate by 2 . The output from the high-pass filter then corresponds to the wavelet coefficients and the output from the low-pass filter is passed on to the next stage of subband splitting. (See [3, Chap. 3] for details and note that his notation-which we use here-differs from that of [4] that we also cite extensively.)

For perfect reconstruction, the synthesis filters $g(n)$ and $h(n)$ corresponding to the decomposition filters $\bar{h}(n)$ and $\bar{g}(n)$ must satisfy certain straightforward conditions. That is to say, they are so-called quadrature mirror filters $[4,31]$ or QMFs. These conditions yield the two equations [3, p. 59]:

$$
\begin{aligned}
& \phi(t)=2 \sum_{n} g(n) \phi(2 t-n), \\
& \psi(t)=2 \sum_{n} h(n) \phi(2 t-n),
\end{aligned}
$$

which are the scaling and wavelet equations, respectively. The reader is cautioned not to confuse the $h(n)$ in (7) with the impulse response of the SUT. (This notation for QMFs is so entrenched that it would potentially be even more confusing to change it. We attempt to minimize ambiguity in following sections by reserving $h($ ) to refer to the system under test except where explicitly stated.)

In Eqs. (6) and (7), the factor of 2 is a scaling or normalizing term. Different normalizations are possible-for example, see [4, p. 59]—and are frequently seen in the literature.

We now make some important observations on orthogonality, not only of wavelets but the coefficients of their associated scaling and wavelet equations too. According to [4, Theorem 3, p. 53], if $\phi(t)$ is an $L^{2} \cap L^{1}$ solution to the scaling equation (6) satisfying the QMF conditions, and $\phi(t)$ is orthogonal for integer translations $k$, so that

$$
\int \phi(t) \phi(t-k) d t \propto \delta(k)
$$

then

$$
\sum_{n} g(n) g(n-2 k) \propto \delta(k)
$$

But (8) is the form of an inner product for the discrete-time case (i.e., a dot product of two sequences rather than an integral for the continuous-time case). Hence, the scaling coefficients have an orthogonal inner product, but only with a step size of 2 or multiples of 2 , although the larger the step size, the greater the chance that there is no overlap with highly-localized signals. For scaling/wavelet coefficients satisfying the QMF filter conditions, the wavelet is orthogonal to the scaling function at the same scale [4, Theorem 17, p. 58], and a similar condition to (8) is satisfied by the $h(n)$ 's.

\section{Source-filter model}

In this section, we first derive the DPWT of the output of the source-filter model when its input is an orthogonal wavelet, before interpreting the result in terms of system identification. 


\subsection{Wavelets as source signals}

For a source-filter model, the observed signal is the convolution of the source signal $x(t)$ and the filter impulse response $h(t)$ :

$$
y(t)=\int x(\tau) h(t-\tau) d \tau
$$

if the filter is time-invariant.

The discrete parameter wavelet transform of $y(t)$ is then

$$
a_{k, m}=\iint \psi_{k, m}(t) x(\tau) h(t-\tau) d \tau d t .
$$

Since convolution is commutative,

$$
\begin{aligned}
a_{k, m} & =\iint \psi_{k, m}(t) h(\tau) x(t-\tau) d \tau d t \\
& =\int h(\tau) \int \psi_{k, m}(t) x(t-\tau) d t d \tau .
\end{aligned}
$$

Following Section 2, let us now choose the source (or 'test') signal to have the same functional form as $\psi_{k, m}$, i.e., $x(t)=\psi_{\alpha, \beta}(t)$. Then the coefficients become

$$
\begin{aligned}
a_{k, m} & =\int h(\tau) \int \psi_{k, m}(t) \psi_{\alpha, \beta}(t-\tau) d t d \tau \\
& =\int h(\tau) \int \psi_{k, m}(t) \psi_{\alpha, d(\alpha, \beta, \tau)}(t) d t d \tau,
\end{aligned}
$$

where $d(\alpha, \beta, \tau)$ is the function that relates the scale and translation indices of the wavelet to the time shift $\tau$ and $h(\tau)$ is now the desired impulse response of the SUT. Introduction of such a function is necessary to yield an integer index when there is a dependence on the continuous variable $\tau$. Note that we must retain the scale index $\alpha$ as an argument in this function in case compression/dilation is related to the scale index (e.g., as in dyadic sampling).

If the family of wavelets is orthogonal, then

$$
a_{k, m}=C \int h(\tau) \delta(k-\alpha) \delta(m-d(\alpha, \beta, \tau)) d \tau .
$$

Here the reader is warned against interpreting both deltas as Kroneckers, as when Eq. (5) was obtained from Eq. (4). In fact, the first delta is a Kronecker by virtue of its arguments $k$ and $\alpha$, which are both discrete. However, the second delta involves the continuous time-shift variable $\tau$. Since the integration is with respect to $\tau$, this must be interpreted as a Dirac delta.

Because $\alpha$ is well chosen to be equal to $k$ (see above), we can make the Kronecker delta equal to one:

$$
a_{k, m}=C \int h(\tau) \delta(m-d(k, \beta, \tau)) d \tau .
$$

For dyadic sampling, $\psi_{k, m}(t)$ is derived from the mother wavelet as

$$
\psi_{k, m}(t)=B \psi\left(2^{k} t+m\right) \quad \text { with } \psi_{0,0}(t)=\psi(t),
$$

where $B$ is a constant. The wavelet coefficients $a_{k, m}$ become

$$
a_{k, m}=A \int h(\tau) \delta\left(m-\left(2^{k} \tau+\beta\right)\right) d \tau,
$$

where $A=B C$. Since $\tau=(m-\beta) / 2^{k}$ yields a zero argument for the Dirac delta, then by the sifting property:

$$
a_{k, m}=A h\left(\frac{m-\beta}{2^{k}}\right) .
$$




\subsection{Interpretation}

We can interpret the DPWT coefficients $a_{k, m}$ in Eq. (9) for fixed $k$ as samples of the SUT impulse response, but scaled and resampled at $2^{-k}$ times some original sampling frequency. Without loss of generality, we can consider this original sampling frequency to be such that the corresponding Nyquist frequency is normalized to 1 . In particular, the impulse response can be expressed in terms of wavelet coefficients as

$$
h\left(\frac{m}{2^{k}}\right)=\frac{a_{k, m+\beta}}{A} .
$$

If $k<0$, then the impulse response is decimated by $2^{|k|}$. For example, if $k=-1$ and $S$ coefficients are computed, the impulse response values are (setting $\beta=0$ for simplicity):

$$
\left\{h(0)=\frac{a_{-1,0}}{A}, h(2)=\frac{a_{-1,1}}{A}, h(4)=\frac{a_{-1,2}}{A}, h(6)=\frac{a_{-1,3}}{A}, \cdots, h(2 S)=\frac{a_{-1, S}}{A}\right\} .
$$

Thus, aliasing can occur if $k$ is not well chosen. Alternatively, if $k>0$, then it is the sequence of wavelet coefficient values (rather than the impulse response) that is decimated. For example, if $k=1$, the impulse response values are (again with $\beta=0$ ):

$$
\left\{h(0)=\frac{a_{1,0}}{A}, h(1)=\frac{a_{1,2}}{A}, h(2)=\frac{a_{1,4}}{A}, h(3)=\frac{a_{1,6}}{A}, \cdots, h\left(\frac{\zeta}{2}\right)=\frac{a_{1, \zeta}}{A}\right\},
$$

where $\zeta=2\lfloor S / 2\rfloor$. A minor concern here is that many wavelet coefficients are not used to determine the impulse response, wasting computation time and memory somewhat. In addition, since only the finite number $S$ of wavelet coefficients were obtained in practice, to achieve a good estimate of the impulse response, the latter should decay to zero over $2^{-k} S$ samples. This sequence is much shorter than the number of available samples $S$, again wasting resources. It seems that the best choice of $k=\alpha$ is zero. In some sense, this is intuitively evident since it corresponds to choice of the mother wavelet as the source signal.

With $k=0$, the impulse response is

$$
h(m)=\frac{a_{0, m+\beta}}{A} \text {. }
$$

Then, the frequency characteristic of the SUT is simply the discrete Fourier transform (DFT) of the DPWT coefficients for $k=0$ :

$$
H(n)=\frac{1}{A} \sum_{m=0}^{S-1} a_{0, m+\beta} e^{-2 \pi j m n / S} .
$$

In summary, an attractive approach to system identification is to excite the SUT with some time-compact function satisfying the orthogonality condition (5), whereupon the system impulse (or frequency) response should be recoverable from the coefficients of the wavelet transform of the output, using the form of the input excitation as the wavelet transform analyzing function.

\section{Discrete-time signals}

For computer processing, all signals are discrete in time and the discrete parameter wavelet transform is replaced by the discrete wavelet transform (DWT). Assume that we have a sampled signal $\{y(n)\}$ of the output of a SUT with discrete impulse response $\{h(n)\}$ excited by a discrete-time input signal $\left\{\psi_{k, m}(n)\right\}$ satisfying the conditions of the previous paragraph. (Henceforth, we will drop the braces \{\} intended to distinguish a sequence from a sample point of that sequence, since no ambiguity should arise.) The continuous analysis above carries over to the discrete case as follows:

DWT synthesis: $\quad y(n)=\sum_{k, m} a_{k, m} \psi_{k, m}(n)$,
DWT analysis: $\quad a_{k, m}=\sum_{n} y(n) \psi_{k, m}(n)$,

Scaling (dyadic): $\quad \psi_{k, m}(n)=B \psi\left(2^{k} n+m\right)$. 
The coefficients of the DWT are given by the discrete wavelet version of Eq. (9), which has precisely the same form as before:

$$
a_{k, m}=A h\left(\frac{m-\beta}{2^{k}}\right)
$$

and by the previous argument, $k=0$ is the best choice to prevent aliasing without wasting resources. Thus, the SUT impulse response in terms of the wavelet coefficients at $k=0$ is as in Eq. (10) and the discrete-time Fourier transform of the impulse function $h()$ can be specified as in Eq. (11). These results are independent of the particular form of the excitation only provided it satisfies the necessary conditions detailed above. This means that we can use not only discrete-time wavelets as inputs, but also their scaling and/or wavelet coefficient values. The scaling condition for multiresolution can also be dropped since only one level (i.e., $k=0$ ) is used.

Unfortunately, as we anticipate from Section 2, the orthogonality condition does not translate so simply and directly from the continuous- to the discrete-time case. Rather than the inner product being zero for all integer translates (as for orthogonal wavelets in the continuous-time case), it is zero for an even step size (e.g., 2).

It is instructive to show at the outset that no discrete-time wavelet can be orthogonal with a step size of 1 . In fact, only a Kronecker delta can fulfill this condition. Let $\varphi_{k, 0}=\left\{w_{k, 0}, w_{k, 1}, \ldots, w_{k, L-1}\right\}$ be the sample sequence of a discrete-time orthogonal wavelet with length $L$, which is not a Kronecker delta. Both $w_{k, 0}$ and $w_{k, L-1}$ are non-zero, otherwise the wavelet is described by a shorter sequence. Since $\varphi_{k, 0}$ is not a Kronecker delta, $L>1$. For orthogonality with step size 1 , the inner product $\left\langle\varphi_{k, m}, \varphi_{k, m+\beta}\right\rangle$ should be 0 for all $k, m$, and $\beta$ except $\beta=0$. However,

$$
\left\langle\varphi_{k, 0}, \varphi_{k, L-1}\right\rangle=w_{k, 0} w_{k, L-1} \neq 0 .
$$

Hence, by contradiction, there does not exist any discrete-time wavelet with compact time support that is orthogonal with a step size of 1 .

\section{System identification using the orthogonality condition}

Given the background above, system identification using the orthogonality of inner products computed by the DWT is relatively straightforward in principle. As depicted in Fig. 1, the SUT is excited by some appropriate input (which we can think of as having $k=0$ ) to produce output sequence $y(n)$. We then take the DWT of the output signal using the input itself as the analyzing function. Then according to the discrete-time version of Eq. (9), the system impulse response (and hence the frequency response) can be estimated directly from the DWT coefficients, $a_{0, m}$.

Although straightforward in principle, some complication arises because the orthogonality condition required to find the DWT coefficients in discrete-time holds only for an even step size in the case of scaling and wavelet coefficients. So, the output sequence from the DWT has only half as many points as its input. In effect, then, we have a multirate problem meaning that considerable care must be taken with possible frequency aliasing [32,33]. The problem is more severe for simulations, as here, than for practical system identification because simulation requires that we generate an appropriate $h(n)$ for the SUT, and generating this $h(n)$ correctly depends on understanding the multiple sampling rates involved.

Hence, we must describe the SUT in a way that is appropriate for system identification after sample-rate decimation by the DWT has occurred. First, an $h(n)$ for the SUT must be generated and, in principle, its system response $H(n)$ could then be found using the DFT. We could then compute the output sequence $y(n)$ by taking the inverse DFT and convolving with the input sequence $x(n)$. But instead of using the baseband representation of $H(n)$ over the range 0 to $f_{s}$ for this purpose, we would need to use its image over the range 0 to $2 f_{s}$. This corresponds to interpolating the $h(n)$

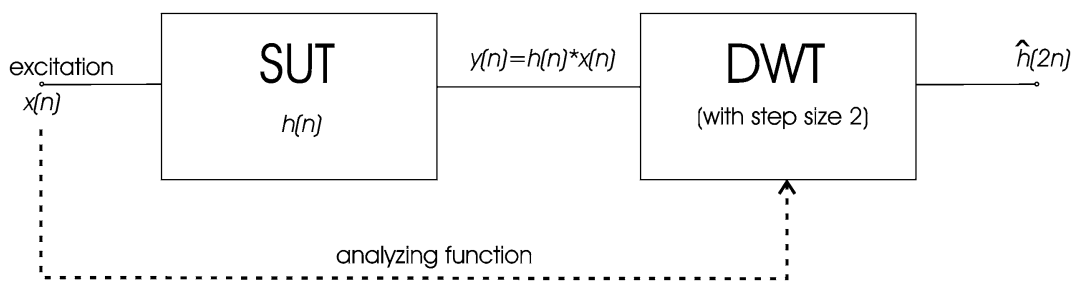

Fig. 1. Schematic diagram of proposed system identification method with a step size of 2 in the discrete wavelet transform. 
sequence by placing zeros between every pair of original sample points [32,33], which is what we actually do. Now, after the decimation at the DWT stage, the SUT's impulse response and corresponding system function are properly represented at a folding frequency of half of $2 f_{s}$, i.e., at $f_{s}$, as required. Note that no anti-alias filtering is required (as used in standard sample-rate conversion) since the sample-rate interpolation is immediately followed by sample-rate decimation by the DFT. In effect, the system identification is obtained at half the number of sample points. This simple scheme is effective for low-pass, band-pass, and high-pass SUTs.

Regarding the time complexity of the new method, it requires just a single inner product computation of two vectors to compute $\hat{h}(2 n)$ (Fig. 1). One vector stores the wavelet coefficients and the other stores the output signal of the SUT. If one vector has $n$ elements and the other has $m$ elements, then the time complexity of the inner product computation is $O(m n)$ since there are $m \times n$ multiplications, $m(n-1)$ additions and $m$ assignments. But since the number of scaling coefficients is very low for the excitations used here (just 4 for Daubechies D4), $m$ can be considered to be a small constant, and the time complexity of the identification is effectively $O(n)$. In practice, computation for our simulations using the new method is almost instantaneous.

\section{Simulations}

We have carried out various simulations to verify the utility of the new method.

\subsection{Choice of excitation}

Using the new method, we attempted to identify the system response of a Chebyshev, IIR, 10th-order high-pass filter with $20 \mathrm{~dB}$ ripple using three different excitations:

(1) Haar wavelet $H_{0,0}(n)= \begin{cases}\frac{1}{\sqrt{2}}, & n=0, \\ -\frac{1}{\sqrt{2}}, & n=1, \\ 0, & \text { otherwise, }\end{cases}$

(2) Daubechies D4 $\left\{\frac{1-\sqrt{3}}{4 \sqrt{2}},-\frac{3-\sqrt{3}}{4 \sqrt{2}}, \frac{3-\sqrt{3}}{4 \sqrt{2}},-\frac{1-\sqrt{3}}{4 \sqrt{2}}\right\}$,

(3) Shannon (truncated sinc).

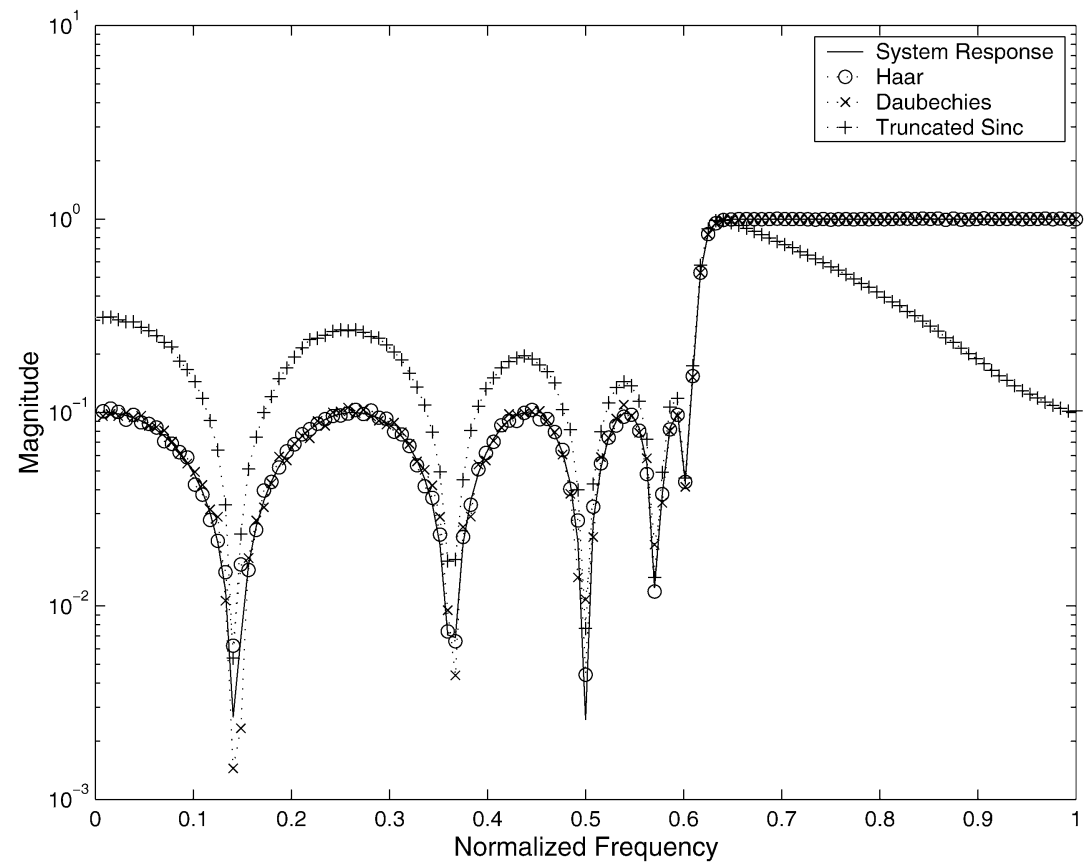

Fig. 2. Frequency response of Chebyshev high-pass filter by the new method using a variety of wavelet scaling coefficients as excitation. 


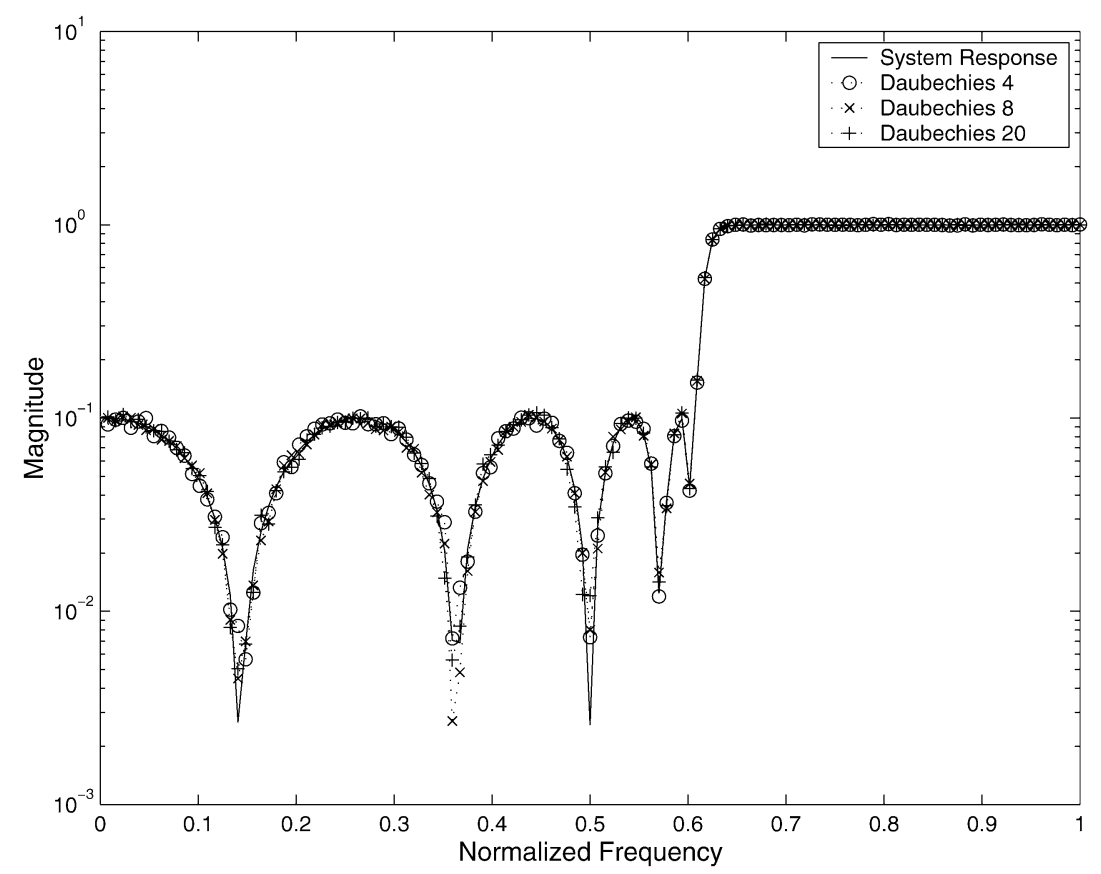

(a)

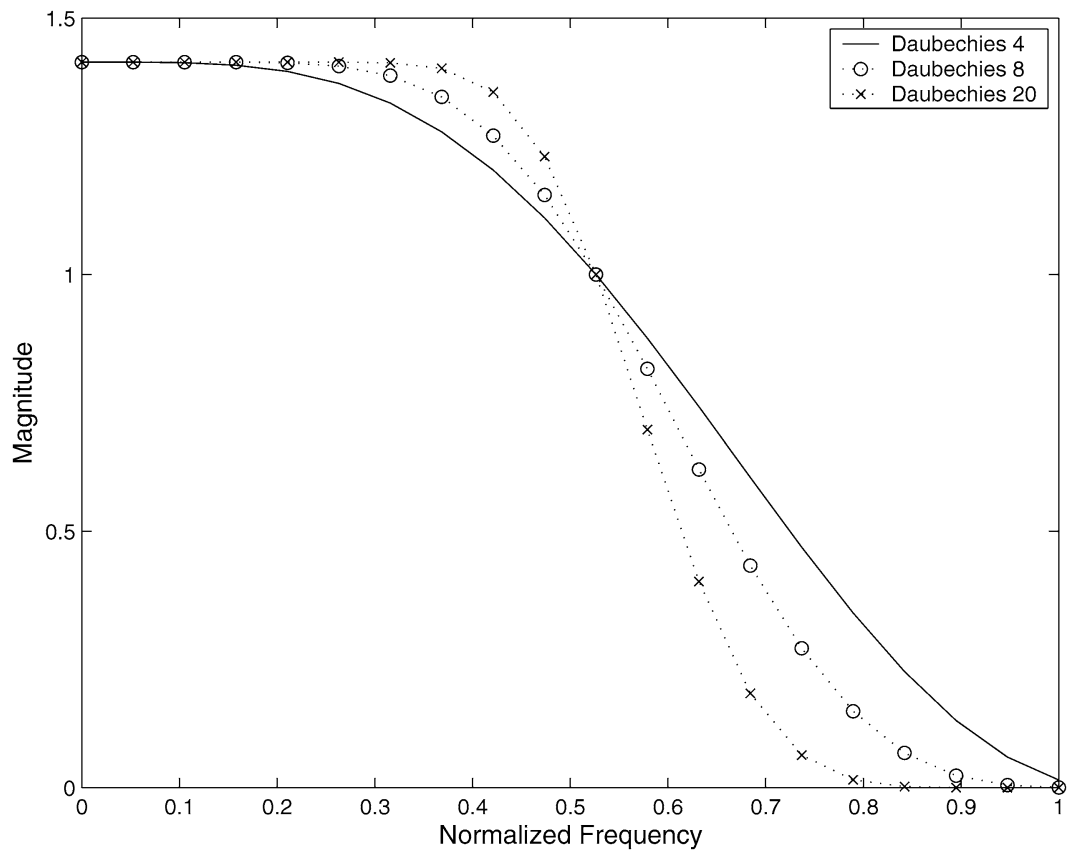

(b)

Fig. 3. (a) Frequency response of Chebyshev high-pass filter by the new method using Daubechies scaling coefficients as excitation with 4,8 , and 20 coefficients. (b) Frequency spectra of these three excitations.

Results are shown in Fig. 2 using a 256-point fast Fourier transform (FFT), so that the system response is identified at 128 points. As can be seen, the Haar and Daubechies excitations give very good identification, but the truncated sinc is unsatisfactory, as it is not orthogonal with a step size of 2.

Figure 3a shows the effect of varying the number of scaling coefficients in the Daubechies excitation. It seems that 4 coefficients is no poorer than either 8 or 20. The spectra of these (low-pass) excitations are shown in Fig. 3b: 
the frequency roll-off increases with the number of coefficients. As rather little seems to be gained by using a higher number of coefficients, the excitation for all subsequent identifications depicted in this paper is the Daubechies D4 scaling coefficients. Before proceeding, the reader should note that identifications were not always done on the same machine so that some very minor discrepancies can be seen between what is nominally the same identification using the same (D4) excitation. These discrepancies are only seen at very low troughs of the system response, effectively below the noise level.

\subsection{Results for different systems}

Although we have examined identification of a variety of frequency-response types, we concentrate here mainly on band-stop system responses, since these are harder to characterize than either low-pass or high-pass, with both finite and infinite impulse responses. In all cases, the stop band was between 0.4 and 0.8 in normalized frequency. Comparisons are made with conventional linear time-invariant system identification tools. In those instances where a band-stop filter does not highlight differences between techniques especially well, we will choose a harder problem to illustrate the advantages of our method.

System identification by wavelet transform has been carried out for the following filter types:

(1) FIR, 50 coefficients;

(2) Butterworth IIR, 10th-order;

(3) Chebyshev IIR, 10th-order with $20 \mathrm{~dB}$ ripple;

(4) Elliptic IIR, 10th-order

using a range of different wavelets and scaling coefficients.

We have deliberately chosen to present simulation results for a wide range of different filters to provide a stringent test of the new method. It is important to include IIR systems because their identification is generally more difficult than for the case of FIR systems. Chebyshev filters are more difficult to identify because they have a sharper cut-off than Butterworth filters. The elliptic filter has significant ripple in the pass and stop bands, which we expect to cause difficulties not encountered with the other filters.

(a)

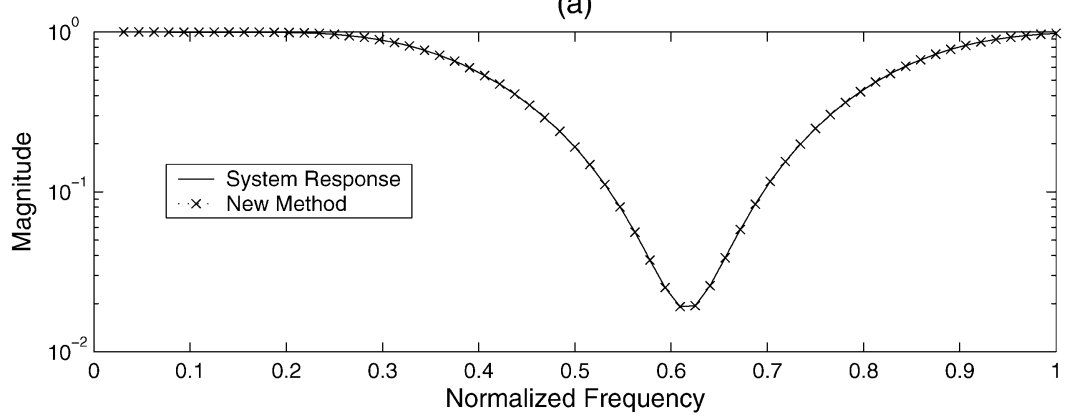

(b)

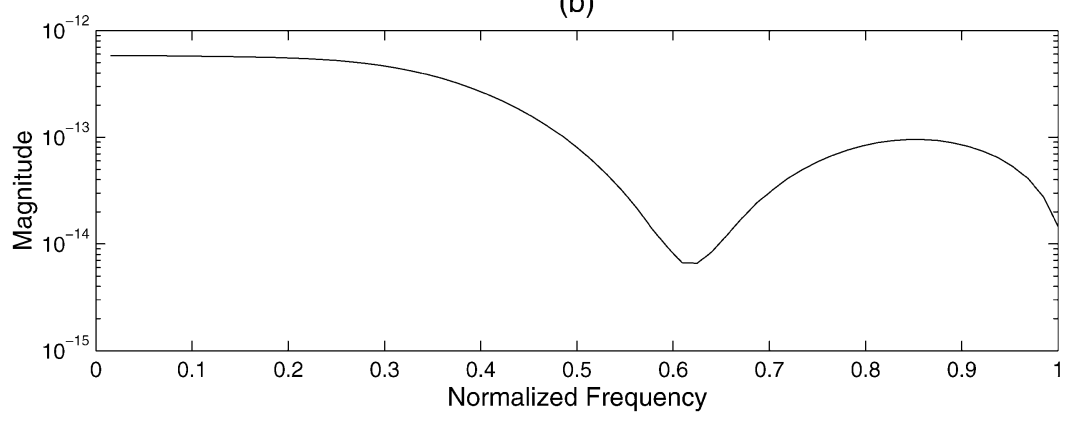

Fig. 4. (a) Frequency response of FIR band-stop filter with the frequency response identified by the new method using D4 scaling coefficients as excitation. (b) The variation of identification error with frequency. 
(a)

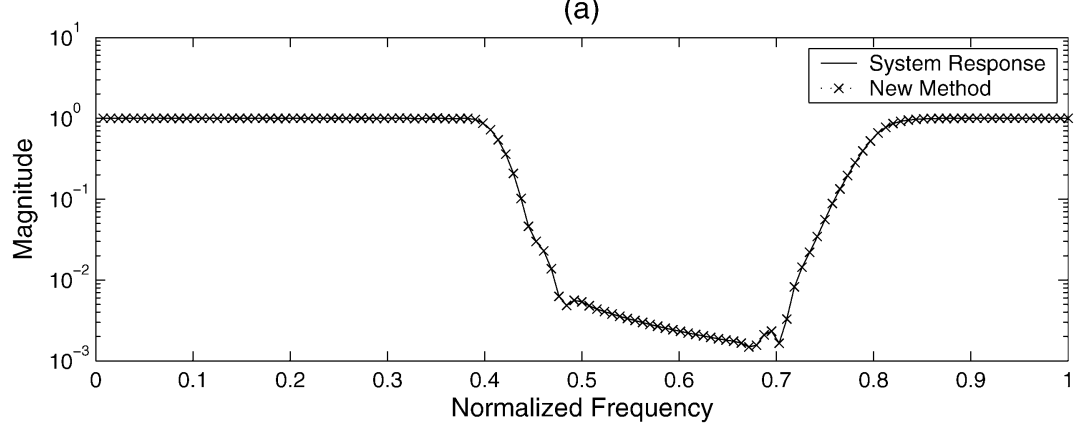

(b)

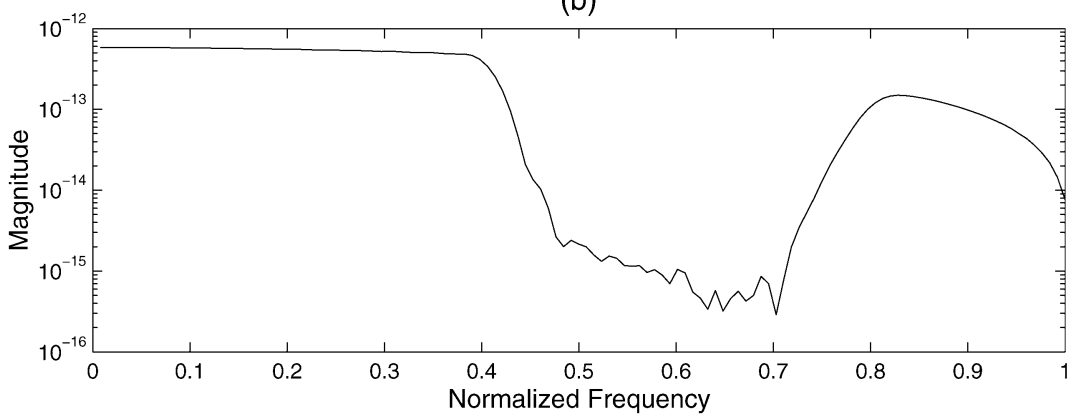

Fig. 5. (a) Frequency response of 10th-order Butterworth band-stop filter with the frequency response identified by the new method using D4 scaling coefficients as excitation. (b) The variation of identification error with frequency.

(a)

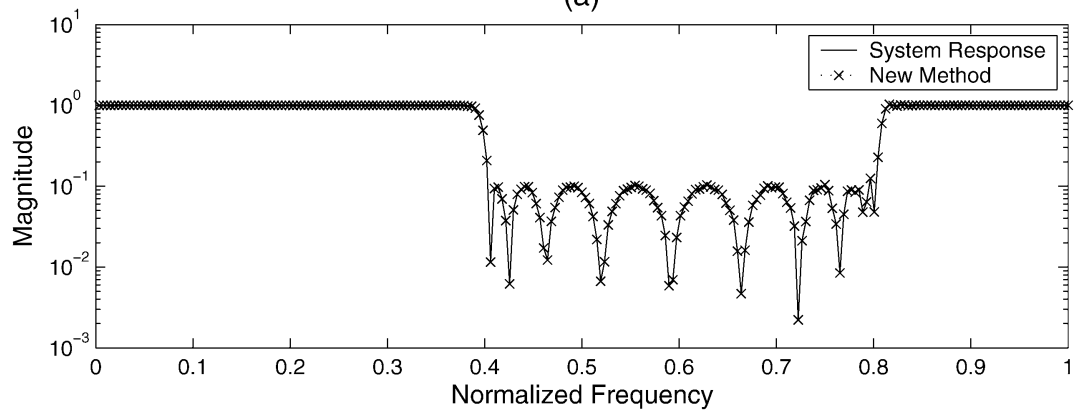

(b)

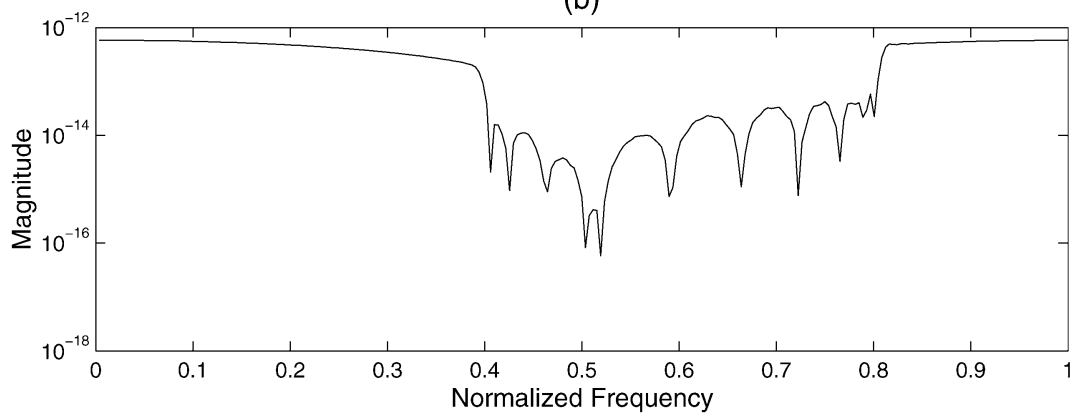

Fig. 6. (a) Frequency response of 10th-order Chebyshev band-stop filter with the frequency response identified by the new method using D4 scaling coefficients as excitation. (b) The variation of identification error with frequency.

Figure 4a shows the system frequency response and the identified frequency response of the FIR band-stop filter identified using the new method. The SUT was simulated using the MATLAB function $\mathrm{fir} 1$, which uses the impulse response windowing method [15, p. 195] with a Hamming window. The identified system response was obtained from 
(a)

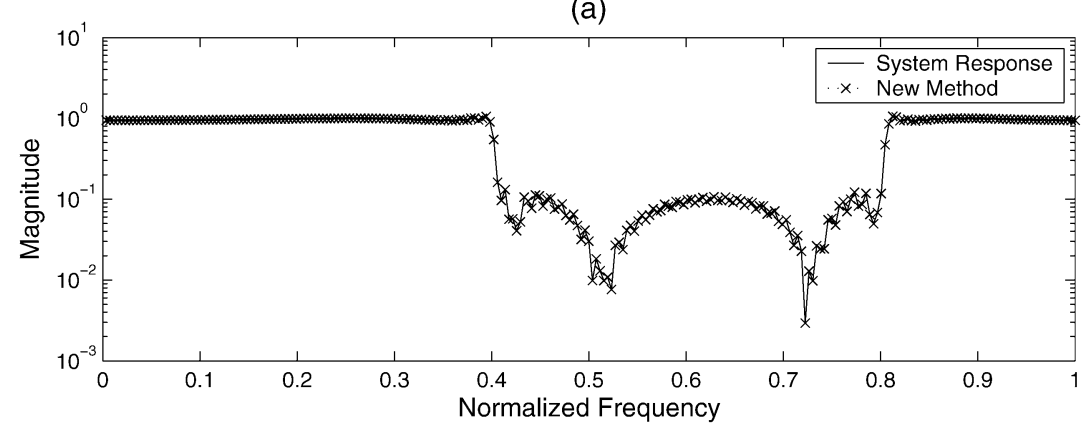

(b)

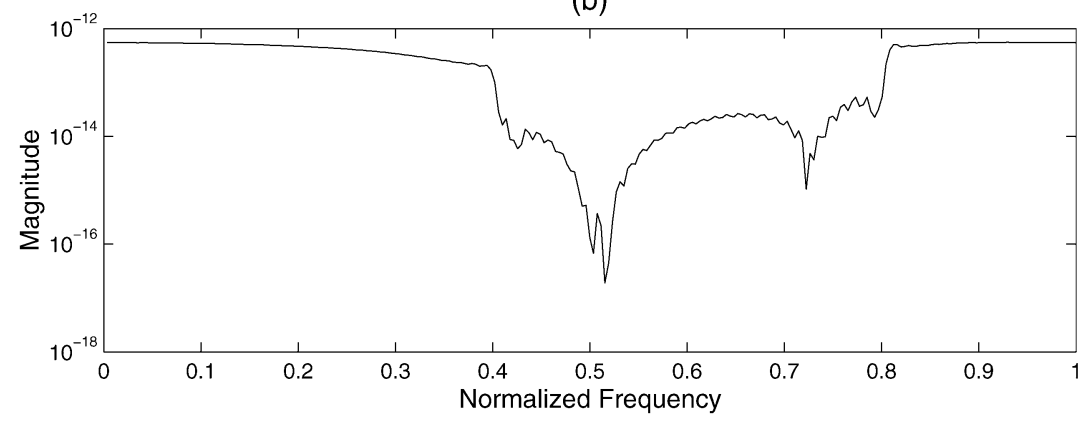

Fig. 7. (a) Frequency response of 10th-order elliptic band-stop filter with the frequency response identified by the new method using D4 scaling coefficients as excitation. (b) The variation of identification error with frequency.

$\hat{h}$ () using a 128-point FFT, giving 64 points shown in the figure. The identification is excellent with errors bounded by $10^{-12}$, being greatest at low frequencies (Fig. $4 \mathrm{~b}$ ).

Figure 5a shows the system frequency response and the identified frequency response of the Butterworth band-stop filter. The SUT was simulated using MATLAB function butter. The identified system response was obtained from $\hat{h}$ () using a 256-point FFT (giving 128 points in the figure). A longer FFT was used for all the IIR filter identifications than for the FIR filter identifications for the obvious reason that the impulse response does not fall to zero and has to be truncated, contributing to the apparent identification error. The error characteristic is similar to the FIR filter, bounded by $10^{-12}$, being greatest at low frequencies (Fig. 5b).

Figure 6a shows the system frequency response and the identified frequency response of the Chebyshev band-stop filter. The SUT was simulated using MATLAB function cheby2. Here the length of the impulse response dictated use of a 512-point FFT, so that the figure shows 256 data points. The identification is similar to the previous filters with a broadly similar pattern of errors, bounded by $10^{-12}$, being greatest away from the stop band (Fig. 6b).

Figure 7 a shows corresponding results for the elliptic band-stop filter. The SUT was simulated using MATLAB function ellip. Again, a 512-point FFT was used, so that the figure shows 256 data points. Again, the identification is very similar to the previous filters with a broadly similar pattern of errors (Fig. 7b).

\section{Comparison with conventional system identification}

In this section, we compare several conventional system identification techniques for the Chebyshev high-pass filter with the new wavelet-based method.

\subsection{Chirp method}

As mentioned in the Introduction, non-parametric identification can be achieved using a wideband excitation such as a frequency chirp (swept sinusoid). Here, the estimated frequency response is obtained as the discrete Fourier transform of the output of the system for such a time-domain input. We used the chirp function in MATLAB as input (see http://www-ccs.ucsd.edu/matlab/toolbox/signal/chirp.html). For low-pass filter responses, this method works well but a chirp signal has poor high frequency content and this affects the identification. 


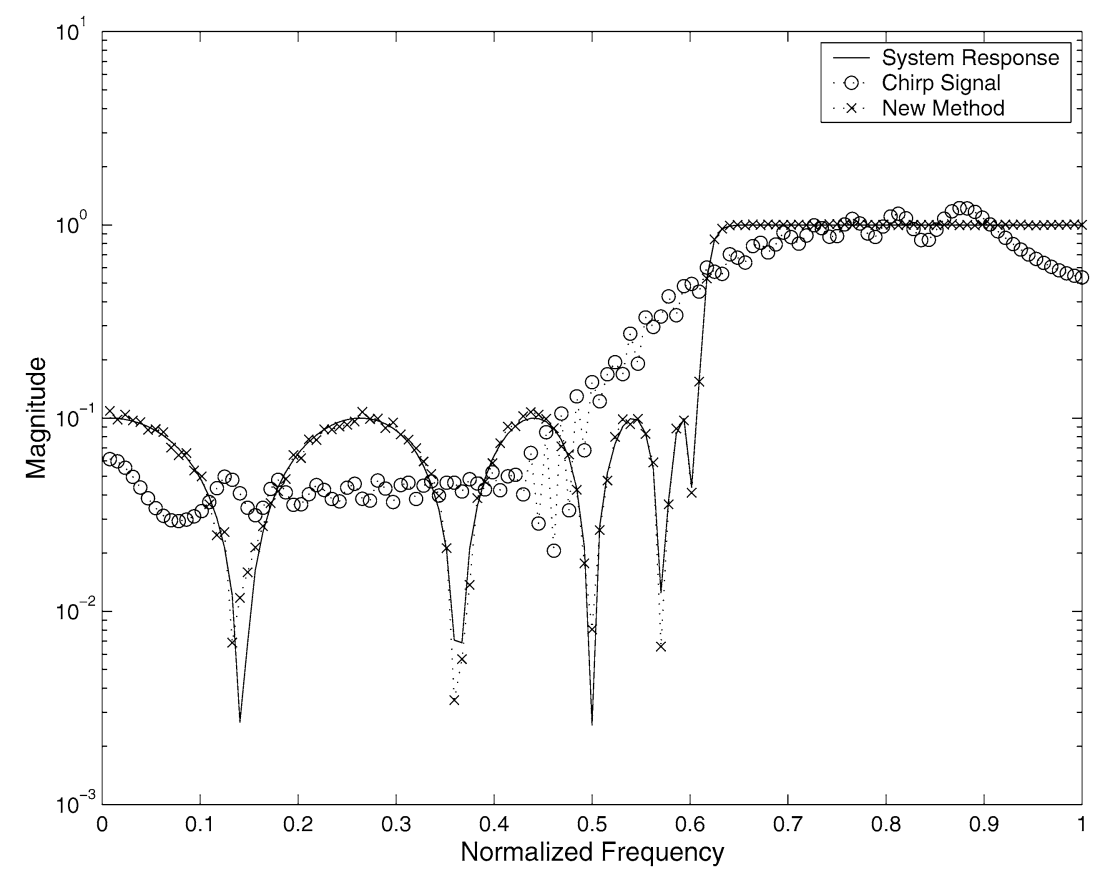

Fig. 8. Frequency response of the Chebyshev high-pass filter identified by wavelet-based method and by DFT in conjunction with a chirp signal excitation.

Figure 8 shows the identification of the Chebyshev high-pass filter response by the wavelet-based method using a 256-point FFT and by using the DFT and a chirp signal. The superiority of the wavelet identification is easily apparent.

\subsection{Time-domain recursion}

Figure 9 shows the comparison of identified frequency responses of the Chebyshev high-pass filter identified by the wavelet-based method and by time-domain recursion using Eq. (1). It is clear that the unbounded nature of the time-domain recursion has led to very substantial errors that are not apparent in the wavelet results.

It might appear from Fig. 9 that the time-domain recursion is effectively useless as a method of finding the (frequency-domain) system function. Note, however, that the identification of the impulse response in the time domain is quite reasonable at first; the errors accumulate over time.

\subsection{Inverse filtering}

As outlined in the Introduction, system identification by inverse filtering requires that we compute the (pseudo)inverse of $X(z)$. Here we work in the time domain. If the system is assumed casual, then the form of the $(p+1) \times(s+1)$ matrix is [24]:

$$
\mathbf{X}=\left[\begin{array}{cccccc}
x(0) & x(1) & x(2) & \ldots & x(s-1) & x(s) \\
0 & x(0) & x(1) & \ldots & x(s-2) & x(s-1) \\
\vdots & \vdots & \vdots & \vdots & \vdots & \vdots \\
0 & 0 & 0 & \ldots & x(0) & x(s-p+1) \\
0 & 0 & 0 & \ldots & 0 & x(s-p)
\end{array}\right]
$$

where the output sequence has $s+1$ samples and the impulse response is estimated at $p+1$ points with $p=s$ (because we can do no better than to estimate $\hat{h}$ () at every available sample point).

The pseudoinverse is found by singular value decomposition [34] using MATLAB function svd. The input excitation for the SVD identification was a pseudorandom sequence of length 1000 samples (truncated to 512 points for subsequent FFT processing to find the system function). SVD minimizes the squared error between the output 


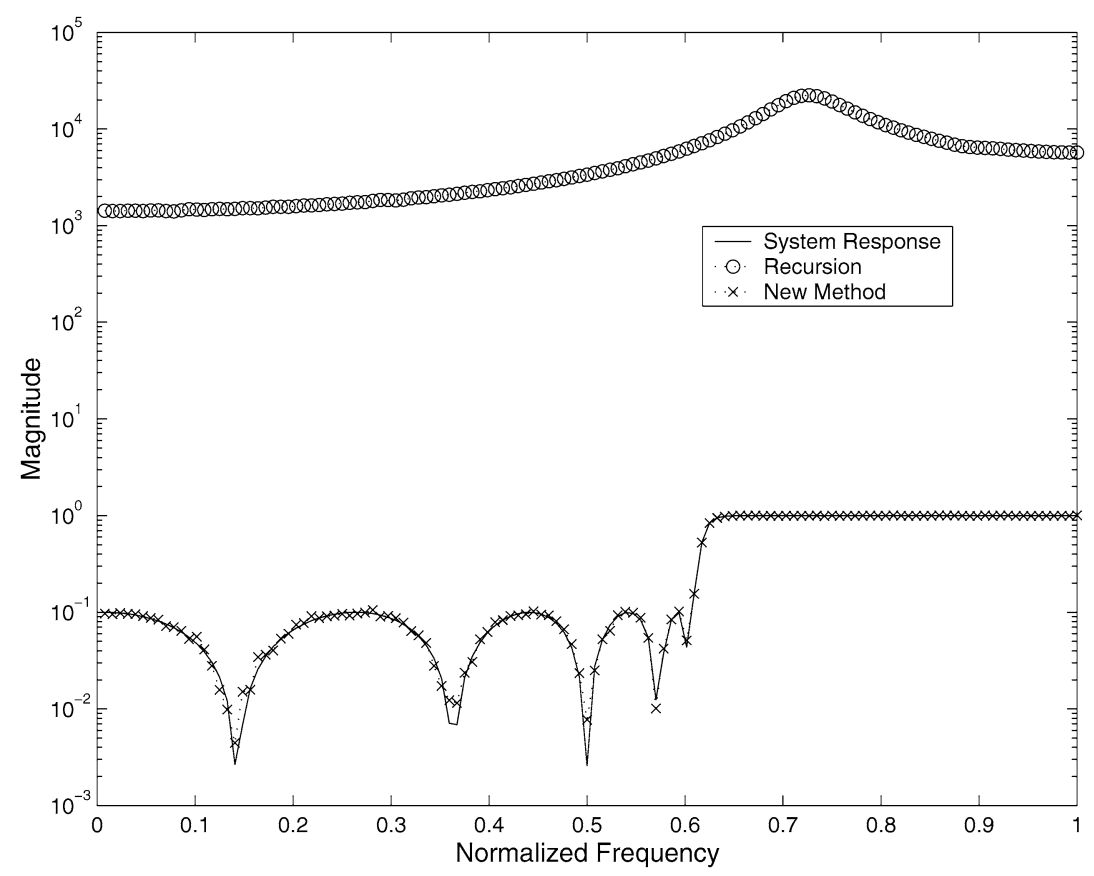

Fig. 9. Frequency response of the Chebyshev high-pass filter identified by the new wavelet-based method and by time-domain recursion.

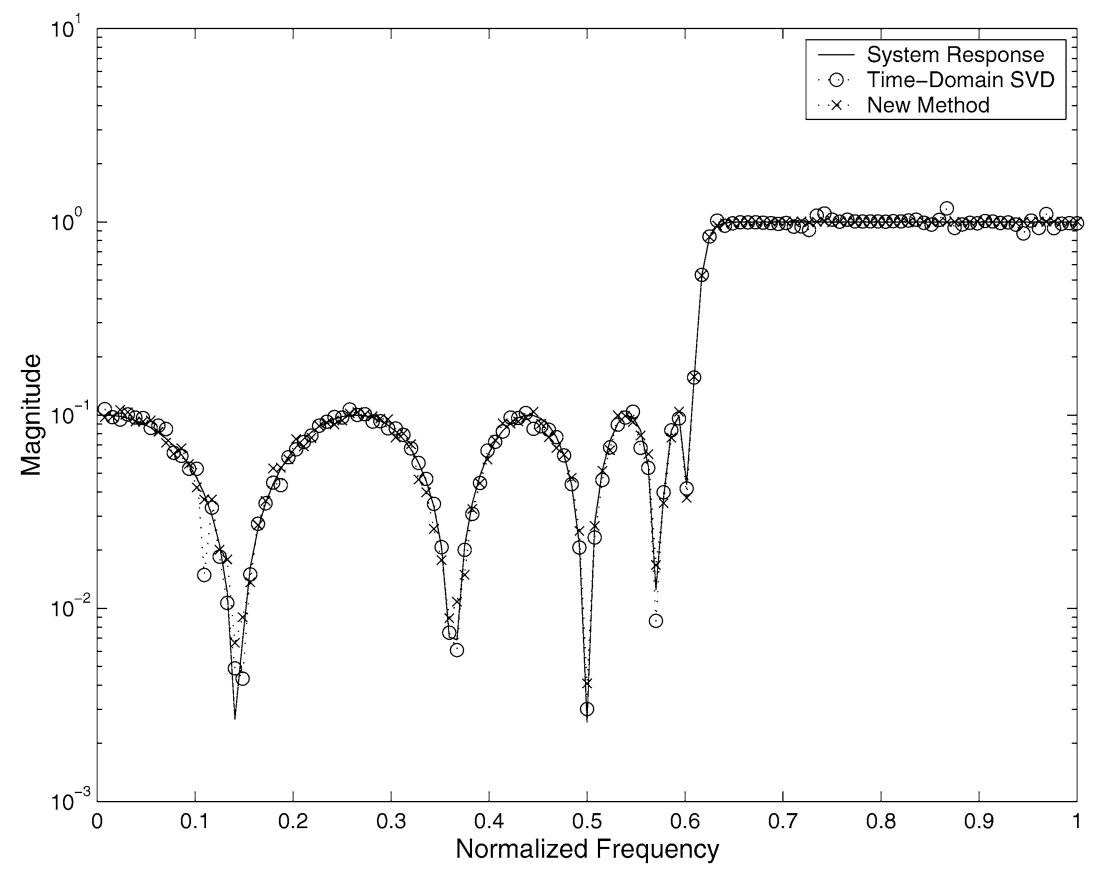

Fig. 10. Frequency response of the Chebyshev high-pass filter identified by the new wavelet-based method and SVD.

sequence and the convolution of the input with the identified system response. Figure 10 shows the comparison of the new method with time-domain SVD for our example Chebyshev high-pass filter. Here, the wavelet-based method outperforms SVD for identification in the pass band and remains reasonable provided we do not extend too far into the stop band. The SVD used here reduces its rank based on discarding $1 \%$ of the data variance (comparable to the added noise). SVD identification could be made as good as the wavelet-based method by using the full rank of the 


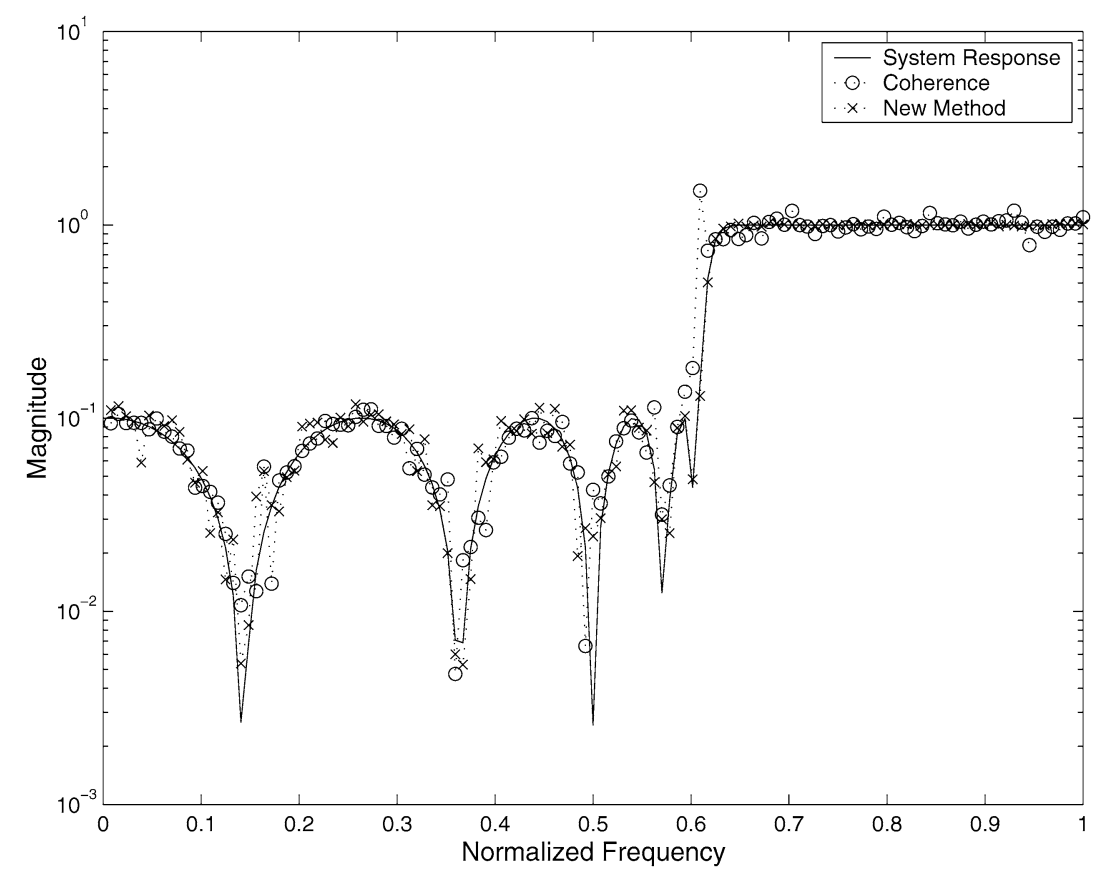

Fig. 11. Frequency response of the Chebyshev high-pass filter identified by the new wavelet-based method and by coherence.

matrices. However, this takes quite a long time to perform (several minutes with a Sun Ultrasparc as opposed to less than a second for the new method).

\subsection{Coherence}

Finally, we have used Eq. (2) to identify the Chebyshev high-pass filter using MATLAB function tfe (transfer function estimate) from the signal processing toolbox. The input excitation for the coherence identification was a pseudorandom sequence of 10,000 samples (truncated to 512 points for subsequent FFT processing to find the system function). Figure 11 shows the comparative results, with the wavelet-based method again proving superior.

\section{Conclusions}

We have developed and described a new method for non-parametric linear time-invariant system identification based on the discrete wavelet transform (DWT). Identification is achieved using a test excitation to the system under test (SUT) that also acts as the analyzing function for the DWT of the SUT's output. The new method can use as excitation any signal that gives an orthogonal inner product in the DWT at some step size. This step size must be even and so cannot be 1 . We favor a step size of 2 used in conjunction with Daubechies D4 scaling coefficients as excitation, since the latter are compact in time. Since step size cannot be 1 , we confront a multirate problem that means we have to oversample the SUT output.

The new method has been compared with several standard techniques for non-parametric identification, namely chirp excitation, time-domain recursion, inverse filtering (using singular value decomposition to invert the input matrix), and coherence analysis. Identification has been carried out for a variety of finite and infinite impulse response systems. The new wavelet-based method proved to be considerably better than the conventional methods in all cases.

In a practical situation, we would obviously not know in advance what the correct identification should be. Hence, identification should ideally be carried out using a variety of differently-motivated methods making different assumptions about the SUT and the test conditions to validate results. Apart from its intrinsic advantages, the new method described here is valuable in that it adds to the number of identification techniques available for this purpose. 


\section{Acknowledgments}

This research was conducted under project grant A448 from the Hong Kong Polytechnic University while author RID was an Academic Visitor to the Centre for Multimedia Signal Processing in the University's Department of Electronic and Information Engineering. We are grateful to Centre Director Prof. Siu for his encouragement and to the Polytechnic University for its generous support.

\section{References}

[1] I. Daubechies, Orthonormal bases of compactly supported wavelets, Commun. Pure Appl. Math. 41 (1988) 909-996.

[2] S.G. Mallat, A theory for multiresolution signal decomposition: The wavelet representation, IEEE Trans. Pattern Anal. Machine Intell. 11 (7) (1989) 674-693.

[3] Y.T. Chan, Wavelet Basics, Kluwer Academic, Boston, MA, 1995.

[4] C.S. Burrus, R.A. Gopinath, H. Guo, Introduction to Wavelets and Wavelet Transforms: A Primer, Prentice Hall, Upper Saddle River, NJ, 1997.

[5] H. Guo, C.S. Burrus, Wavelet and image compression using the Burrows Wheeler transform and the wavelet transform, in: Proceedings of the IEEE International Conference on Image Processing, ICIP-97, vol. I, Santa Barbara, CA, October 1997, pp. 65-68.

[6] D.L. Donoho, De-noising by soft-thresholding, IEEE Trans. Inform. Theory 41 (3) (1995) 613-627.

[7] S. Del Marco, J. Weiss, Improved transient signal detection using a wave packet-based detector with an extended translation-invariant wavelet transform, IEEE Trans. Signal Process. 45 (4) (1997) 841-850.

[8] N. Erdol, F. Basbug, Wavelet transform based adaptive filtering, in: J. Vandewalle, R. Boite, M. Moone, A. Oosterlinck (Eds.), Signal Processing VI: Theories and Applications, Elsevier, Amsterdam, 1992, pp. 1117-1120.

[9] S. Hosur, A.H. Tewik, Wavelet transform domain LMS algorithm, in: Proceedings of the IEEE International Conference on Acoustics, Speech and Signal Processing, vol. 3, Minneapolis, MN, April 1993, pp. 508-510.

[10] M.K. Tsatsanis, G.B. Giannakis, Time-varying channel equalization using multiresolution analysis, in: Proceedings of the IEEE-SP International Symposium on Time-Frequency Time-Scale Analysis, Victoria, Canada, October 1992, pp. 447-450.

[11] M. Doroslovački, H. Fan, On-line identification of echo-path impulse responses by Haar-wavelet-based adaptive filtering, in: Proceedings of the IEEE International Conference on Acoustics, Speech and Signal Processing, vol. 2, Detroit, MI, 1995, pp. 1065-1068.

[12] X.W. Yang, K. Wang, S.A. Shamma, Auditory representations of acoustic-signals, IEEE Trans. Inform. Theory 38 (2) (1992) $824-839$.

[13] K. Wang, S.A. Shamma, Modeling the auditory functions in the primary cortex, Opt. Eng. 33 (7) (1994) 2143-2150.

[14] R.A. Gopinath, C.S. Burrus, Wavelet transforms and filter banks, in: C.K. Chui (Ed.), Wavelets: A Tutorial in Theory and Applications, Academic Press, San Diego, 1992, pp. 603-655.

[15] R.I. Damper, Introduction to Discrete-Time Signals and Systems, Chapman \& Hall, London, 1995.

[16] L.C. Wood, S. Treitel, Seismic signal processing, Proc. IEEE 63 (1975) 649-661.

[17] J.S. Bendat, A.G. Piersol, Random Data: Analysis and Measurement Procedures, third ed., Wiley, New York, 2000.

[18] A. Juditsky, Q. Zhang, B. Delyon, P.-Y. Glorennec, A. Benveniste, Wavelets in identification, IRISA Publication No. 849, September 1994.

[19] M. Pawlak, Z. Hasiewicz, Nonlinear system identification by the Haar multiresolution analysis, IEEE Trans. Circuits Systems I-Fundam. Theory Appl. 45 (9) (1998) 945-961.

[20] W.J. Staszewski, Identification of non-linear systems using multi-scale ridges and skeletons of the wavelet transform, J. Sound Vibrat. 214 (4) (1998) 639-658.

[21] W.J. Staszewski, Analysis of non-linear systems using wavelets, Proc. Inst. Mech. Eng. C 214 (11) (2000) 1339-1353.

[22] D. Coca, S.A. Billings, Non-linear system identification using wavelet multiresolution models, Int. J. Control 74 (18) (2001) $1718-1736$.

[23] R. Ghanem, F. Romeo, A wavelet-based approach for model and parameter identification of non-linear systems, Int. J. Nonlinear Mech. 36 (5) (2001) 835-859.

[24] A.N. Robertson, K.C. Park, K.F. Alvin, Extraction of impulse response data via wavelet transform for structural system identification, J. Vibrat. Acoust. Trans. ASME-120 (1) (1998) 252-260.

[25] M.K. Tsatanis, G.B. Giannakis, Time-varying system identification and model validation using wavelets, IEEE Trans. Signal Process. 41 (12) (1993) 3512-3523.

[26] M.I. Doroslovački, H. Fan, L. Yao, Wavelet-based identification of linear discrete-time systems: Robustness issues, Automatica 34 (12) (1998) 1637-1640.

[27] Y. Zheng, Z. Lin, D.B.H. Tay, Time-varying parametric system multiresolution identification by wavelets, Int. J. Syst. Sci. 32 (6) (2001) 775-793.

[28] M.I. Doroslovački, H. Fan, Wavelet-based linear system modeling and adaptive filtering, IEEE Trans. Signal Process. 44 (5) (1996) 11561167.

[29] M. Lang, H. Guo, J.E. Odegard, C.S. Burrus, R.O. Wells, Nonlinear processing of a shift-invariant DWT for noise reduction, in: H.H. Szu (Ed.), Wavelet Applications II, in: Proceedings of the SPIE Conference, vol. 2491, SPIE, Orlando, FL, 1995, pp. 640-651.

[30] H. Sari-Sarraf, D. Brzakvoic, A shift-invariant discrete wavelet transform, IEEE Trans. Signal Process. 45 (10) (1997) $2621-2626$.

[31] D. Esteban, C. Galand, Application of quadrature mirror filter to split-band voice coding schemes, in: Proceedings of the IEEE International Conference on Acoustics, Speech, and Signal Processing, ICASSP '77, Hartford, CN, 1977, pp. 191-195.

[32] R.E. Crochiere, L.R. Rabiner, Multirate Digital Signal Processing, Prentice Hall, Englewood Cliffs, NJ, 1983. 
[33] R.E. Crochiere, L.R. Rabiner, Multirate processing of digital signals, in: J.S. Lim, A.V. Oppenheim (Eds.), Advanced Topics in Digital Signal Processing, Prentice Hall, Englewood Cliffs, NJ, 1988, pp. 123-198.

[34] B. Noble, J.W. Daniel, Applied Linear Algebra, Prentice Hall International, London, UK, 1988.

Robert Luk received the BSc and DipEng degrees in electronic engineering from the Department of Electronics and Computer Science at the University of Southampton, England, in 1987, the MSc degree in cognition, computing, and psychology from the University of Warwick, England, in 1989, and then returned to Southampton to complete his PhD in speech synthesis in 1992. Dr. Luk then joined the Department of Chinese, Translation and Linguistics at the City University of Hong Kong as a Lecturer in computational linguistics, and moved to the Department of Computing at Hong Kong Polytechnic University in 1996, where he now works mainly on information retrieval. Dr. Luk's other research interests include natural language processing, tone recognition using wavelets, and formalisms for text-to-speech synthesis. He is a Senior Member of the IEEE.

Robert I. Damper obtained the MSc degree in biophysics in 1973, and the PhD degree in electrical engineering in 1979, both from the University of London. He also holds the Diploma of Imperial College, London, in electrical engineering. He was appointed Lecturer in electronics at the University of Southampton in 1980, Senior Lecturer in 1989, Reader in 1999, and Professor in 2003. His research interests include speech science and speech technology, signal and pattern processing, neural computing, and mobile robotics. Prof. Damper has published more than 270 research articles and authored the undergraduate text Introduction to DiscreteTime Signals and Systems. He is a past Chairman of the IEEE Signal Processing Chapter of the UK and Republic of Ireland Section (1992-1999), a Chartered Engineer and a Chartered Physicist, a Fellow of the UK Institution of Electrical Engineers and of the UK Institute of Physics, a Member of the Acoustical Society of America and of the International Speech Communication Association, and a Senior Member of the IEEE. Prof. Damper serves on the editorial boards of the International Journal of Speech Technology and the International Journal of Information Technology. 